\title{
Short-term effects of tiotropium on the pulmonary function and quality of life (QOL) in patients at risk and with mild chronic obstructive pulmonary disease (COPD)
}

\author{
Tatsuyuki Miyashita, Hideo Oya, Nagato Sato, Koichi Hagiwara, Minoru Kanazawa \\ The Department of Respiratory Medicine, Saitama Medical University
}

\begin{abstract}
Purpose: Although the short-term effects of tiotropium for patients with Stage II-IV COPD have been established, the effects on patients in earlier stages are not known. We studied the short-term effects of tiotropium on pulmonary function, quality of life (QOL), and clinical symptoms in patients at risk for and with mild COPD.

Subjects and methods: The subjects enrolled the study consist of 85 patients who visited the clinics due to respiratory symptoms, were more than 40 years old, and had a smoking history of more than 10 pack-years were enrolled. They were divided into an At risk-Stage I COPD group and a Stages II-IV COPD group. After the administration of tiotropium for 12 weeks, the changes in the pulmonary function test, QOL, and subjective symptoms were compared before and after the therapy.

Results: Tiotropium increased the FEV1, FVC, and IC and improved the SGRQ scores in the patients in the Stage II-IV COPD group, while it did not cause a significant difference in the At risk-Stage I groups. Some patients in the At risk-Stage I group who experienced an improvement in their subjective symptoms.

Conclusions: We could not show that the patients in the At risk-Stage I group benefited from tiotropium. However, some patients in the At risk-Stage I groups showed improvements in their symptoms. Tiotropium may be effective to relieve symptoms in some patients even when they are in the At risk-Stage I groups.
\end{abstract}

Key Words: COPD, bronchodilators, tiotropium, quality of life, symptoms

(Received December 21, 2012; Accepted Jannuary 11, 2013)

\section{Background}

Chronic obstructive pulmonary disease (COPD) is a disease characterized by irreversible airflow limitation that is usually progressive ${ }^{1,2)}$. The COPD patients complain of cough, sputum, and dyspnea that exacerbates after exercise. Most patients die of respiratory failure known as the acute exacerbation of COPD that is elicited by an acute respiratory tract infection. COPD is one of the leading causes of morbidity and mortality worldwide, and is estimated to be the cause of death for 2.75 million people annually, and thus is a serious public health prob$\operatorname{lem}^{3,4)}$

The management procedures of COPD include: (1) inhibition of smoking and avoidance of inhaled irritants, since they are the main causes of chronic airway inflammation; (2) pharmacotherapy and pulmonary rehabilitation to relieve patients' symptoms; (3) long-term home oxygen therapy that has been shown to increase survival

correspondence to: Prof. Minoru Kanazawa, Department of Respiratory Medicine, Saitama Medical University, 38 Morohongo, Moroyama-machi, Saitama 350-0495, Japan. TEL: +81-49-276-1319 Fax:+81-49-276-1635 E-mail: mkanazaw@saitamamed.ac.jp of the patients with severe COPD; and (4) immunization against respiratory pathogens to reduce the chance of acute exacerbation. The goals of management are an improvement in the patients' activity of daily living (ADL) and health-related quality of life (HRQOL) as well as their survival. Among the procedures stated above, it is especially important to quit smoking as it reduces the mortality of the patients not only from COPD but from other pulmonary diseases, cancers of several organs and cardiovascular diseases ${ }^{5}$.

Guidelines for COPD have been published from a variety of groups that include the Global Initiative for Chronic Obstructive Lung Disease (GOLD) ${ }^{2)}$, the American Thoracic Society and European Respiratory Society (ATS/ERS), the Canadian Respiratory Society, and the Japanese Respiratory Society. All guidelines determine the therapeutic interventions based on the severity of the COPD. The patients with moderate COPD are usually treated by long-acting bronchodilators, since these drugs improve QOL and decrease acute exacerbations $^{1,2)}$. Inhaled bronchodilators tend to be preferred by patients to oral tablets, because they provide a greater improvement in the pulmonary function, present less systemic side effects, and thus are better tolerated. 
Tiotropium is one of the most effective long acting bronchodilators $^{1,2,6,7)}$. A post hoc analysis of randomized controlled trials has suggested that the initiation of tiotropium at earlier stages improves pulmonary function and $\mathrm{HRQOL}^{8}$. The administration of tiotropium from earlier stages may be a promising strategy in the management of COPD.

Tiotropium has been shown to be effective for patients with stages II-IV COPD ${ }^{1,2)}$. On the other hand, the effect on earlier stages of COPD has not been well documented. We hypothesized that the administration of tiotropium to patients at risk and with mild COPD would relieve the clinical symptoms, improves the pulmonary functions, and QOL. To test the hypothesis, we conducted a singlearm, prospective clinical study in which tiotropium was administered for 12 weeks to the COPD patients, and the change in their pulmonary function, the health status as measured by the Saint George's Respiratory Questionnaire (SGRQ) ${ }^{9,10)}$, and the subjective change in their clinical symptoms were compared between the At risk - Stage I COPD group and the Stage II-IV COPD group.

\section{Methods}

\section{Subjects}

This study was approved by the ethics committees of the member hospitals of the Saitama Northwest COPD Research Group. Patients who visited member hospitals or clinics of the group between March 2005 and February 2006 complaining of respiratory symptoms, such as chronic cough, sputum and dyspnea on exertion, were invited to enroll if: 1) they were more than 40 years old; and 2) they had smoked more than 10 pack-years. They were excluded if: 1) they had diseases other than COPD that may cause dyspnea; 2) they had a history of asthma; or 3) they had a recent respiratory tract infection. Written informed consent was obtained before any study procedures were undertaken.

\section{Protocol}

The dose of tiotropium (Spiriva(R); Boehringer Ingelheim Pharmaceuticals, Inc., Ridgefield, CT) that was used for daily clinical practice $(18 \mu \mathrm{g} \text { once daily })^{11)}$ was also used in the current study. On the screening visit, the patients underwent pulmonary function testing to classify themselves into the At risk-Stage I group or the Stages II-IV group based on the GOLD guideline ${ }^{2)}$. The patients were evaluated using a pulmonary function test (measured on Weeks 0, 4 and 12), the SGRQ (performed on Weeks 0 and 12) and the inquiry on the change of their clinical symptoms that included cough, sputum and dyspnea (performed on Week 12). The appearance of adverse events was monitored during the entire study period.

\section{Pulmonary function test}

The pulmonary function test was performed using an office spirometer, where the forced expiratory volume in one second (FEV1), forced vital capacity (FVC), and inspiratory capacity (IC) were measured. For each measurement, the values obtained before and $20 \mathrm{~min}$ after an inhalation of a short-acting beta2-agonist (salbutamol $200 \mu \mathrm{g}$ ) were recorded. The values of the pulmonary function tests obtained on Day 0 , immediately before and after the administration of the first dose of tiotropium, were defined as the pre- and post-salbutamol baseline values $^{12)}$.

\section{SGRQ}

The SGRQ contains 50 questions covering 3 domains (symptoms, activity, and impact) each of which were scored from 0 (best health) to 100 (worst health). A 4-point change was considered significant ${ }^{13)}$, and we scored is as equal to or more than 4-point decreases as an improvement, equal to or more than 4-point increases as a worsening, and the scores in-between as a stable condition.

\section{Inquiry on the clinical symptoms}

On Week 12, the patients were asked how their cough, sputum and dyspnea changed over the 12-week treatment period, and they chose the answers from among the categories of improvement, stable symptoms and worsening.

\section{Statistical analysis}

The statistical analyses were performed using the SAS software package (version 9.13). The differences between the pre- and post-treatment values for both the pulmonary function test and the SGRQ were tested by Student's paired t-test. The data were reported as the means \pm standard deviation (SD). P-values less than 0.05 were considered to be statistically significant.

\section{Results}

\section{Subjects}

A total of 85 patients were enrolled, of which 78 completed the study. The characteristics of the patients who completed the study did not show any significant differences between the At risk-Stage I and Stages II-IV groups (Table 1). Seven subjects discontinued: 4 were due to adverse events; 3 did not visit the hospitals or clinics for unknown reasons.

\section{Pulmonary function test}

Our study was performed in the daily clinical setting, where pulmonary function testing that required expensive equipment was not practical. We therefore investi- 
Table 1. Patient characteristics

\begin{tabular}{l|c|c|c}
\hline & At risk-Stage I & Stages II-IV & Overall \\
\hline No. of subjects & 38 & 40 & 78 \\
\hline Male/female & $38 / 0$ & $36 / 0$ & $74 / 4$ \\
\hline Age (years)* & $68.4 \pm 7.9$ & $69.1 \pm 8.0$ & $68.7 \pm 7.9$ \\
\hline Smoking (pack years)* & $53.2 \pm 30.8$ & $52.7 \pm 25.1$ & $52.9 \pm 26.3$ \\
\hline Cough & 28 & 23 & 51 \\
\hline Sputum & 30 & 26 & 56 \\
\hline Dyspnea & 30 & 35 & 65 \\
\hline
\end{tabular}

*Data are presented as the mean \pm SD.

gated the FEV1, FVC, and IC, which are easily measured using a portable spirometer, and nevertheless provide important information on the patients' pulmonary function.

The administration of salbutamol increased the baseline values for FEV1 and FVC for both groups (Figs. 1a and $b$ ). Tiotropium increased the values further in the Stages II-IV patients (Figs. 1a and 1b, right panels) while it did not show a significant difference in the At riskStage I patients (Figs. 1a and b, left panels). The IC did not change in either group (Fig. 1c). We initially thought that the failure to show a significant difference in the FEV1 and FVC in the At risk-Stage I group may have been because of the small number of patients $(n=38)$ enrolled. However, a model calculation showed that, even if we had twice the number of the patients who showed a similar trend in the pulmonary function test, our findings would have fail to show the significance. We therefore concluded that tiotropium scarcely improved the pulmonary function of the patients in the At risk-Stage I groups.

\section{SGRQ}

The patients in the Stage II-IV group showed significant improvement in the SGRQ score after the tiotropium treatment, while those in the At risk-Stage I group did not show any improvement (Fig. 2a). The results of the SGRQ significantly improved in the patients in the Stage II-IV group, while they did not show any difference in the patients in the At risk-Stage I group (Fig. 2a). The patients in the Stage II-IV group presented more improvement in both the activity and impact domains of the SGRQ, while the improvement in the symptom domain was similar for both groups.

\section{Clinical Symptoms}

We focused on the clinical symptoms that include cough, sputum and dyspnea and asked a simple question to the patients as to whether the tiotropium treatment improved their symptoms. The reason we added this question to this study was that we had noticed that tiotropium treatment remarkably improved these symptoms in some patients in the earlier stages of COPD. The result is shown in Figure 3. Although the proportion was smaller in the Stage II-IV group, some of the patients in the At
risk-Stage I group felt that their symptoms were relieved, especially cough and dyspnea. The result shows that there are patients, even in the At risk-Stage I group, who benefit from tiotropium based on improvements in their clinical symptoms.

\section{Adverse events}

The overall incidence of adverse events was $14.0 \%$ (1 patient $(1.2 \%)$ in the At risk-stage I group; 11 patients (12.8\%) in the Stage II-IV group). Three patients (3.5\%) in Stages II-IV discontinued the trial due to probable drug-related adverse events that included ileus, drug allergy, and the aggravation of prostate hypertrophy. One patient $(1.2 \%)$ experienced an acute exacerbation of COPD and dropped out from the study. The most common adverse event was dry mouth (2 patients $(2.3 \%)$ in the At risk-stage I group; 3 patients $(3.5 \%)$ in the Stage II-IV group), although it was mild and none of the patients stopped taking tiotropium. Other adverse events observed were frequent urination (3 patients $(3.5 \%)$ ), upper respiratory tract infection (1 patient $(1.2 \%))$, and dizziness (1 patient (1.2\%)).

\section{Discussion}

It has been shown that tiotropium is effective for patients with moderate to severe COPD ${ }^{1,2)}$. In the current study, we investigated whether patients in the earlier stages of COPD benefit from tiotropium.

We found that the patients in the Stage II-IV group showed a significant improvement in the pulmonary function test, and this is consistent with the results of the previous reports. We could not find any improvement in the pulmonary function test in the patients in the At riskStage I group: even in a model calculation, where the number of the patients was assumed to have doubled, we could not obtain a significant difference. Tiotropium may therefore provide little benefit in the pulmonary function for patients in the At risk-Stage I group.

We also had a negative result for the improvement of the SGRQ score in the patients in the At risk-Stage I group. Our study was performed in an outpatient clinic setting, where we found that most of the patients with At risk-Stage I COPD visit the doctors' office complaining of cough, sputum, or dyspnea. Their social activity is usually well maintained, because their disease is not severe enough to impair it. We thus focused on cough, sputum, and dyspnea, and investigated the change in them after the tiotropium treatment. Some patients showed an improvement. Since the reason of the visit to the office was for such symptoms, we found that many of the patients were satisfied with the treatment. The patients in the At risk-Stage I groups are not at the risk of dying because of COPD. The important thing for these patients is to fully understand the risk of COPD and relieve their 
A

At risk-Stage I

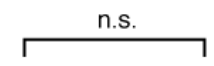

FEV1(L)

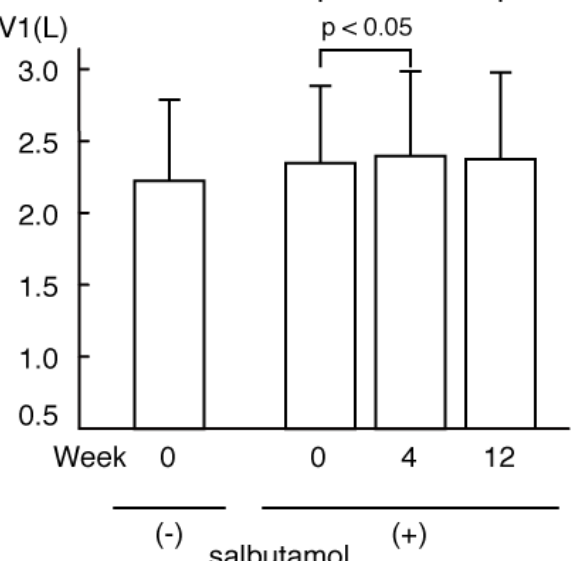

B

At risk-Stage I

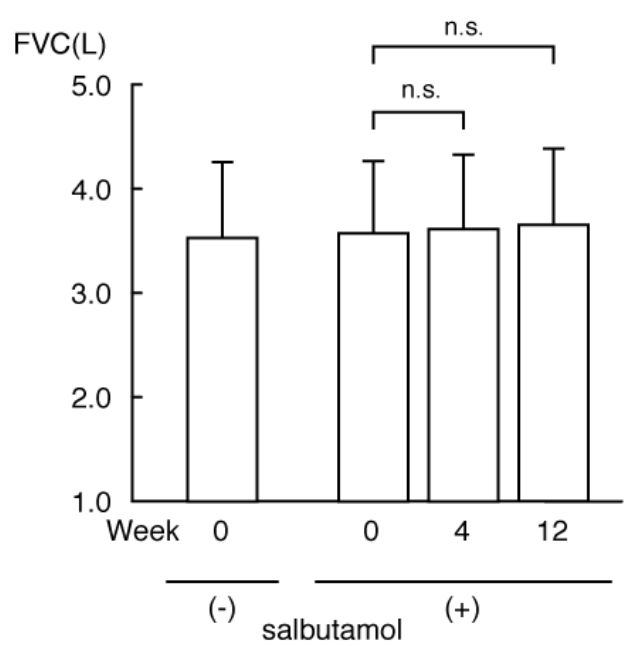

C

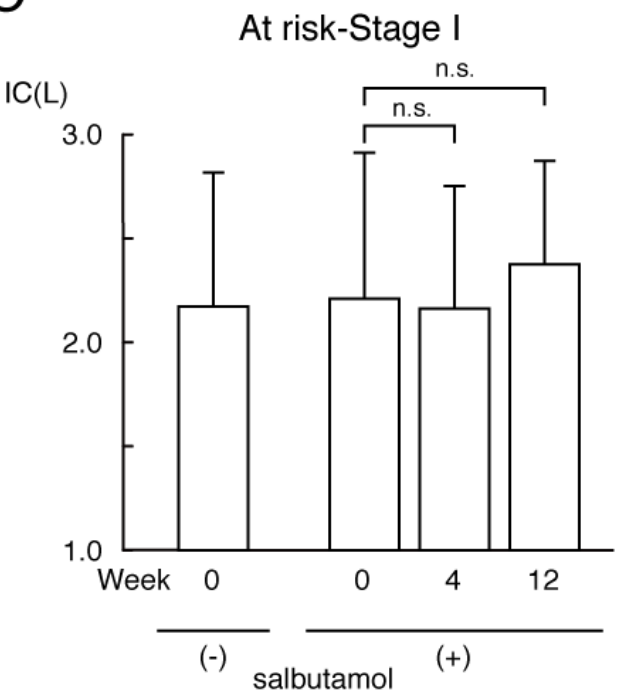

Stages II-IV

FEV1(L)

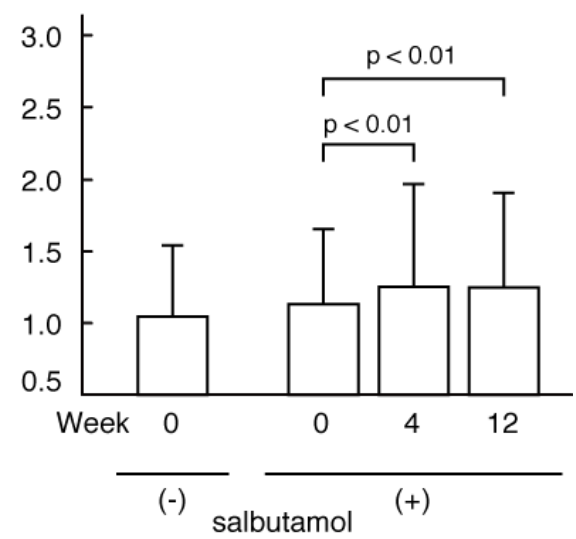

Stages II-IV

FVC(L)

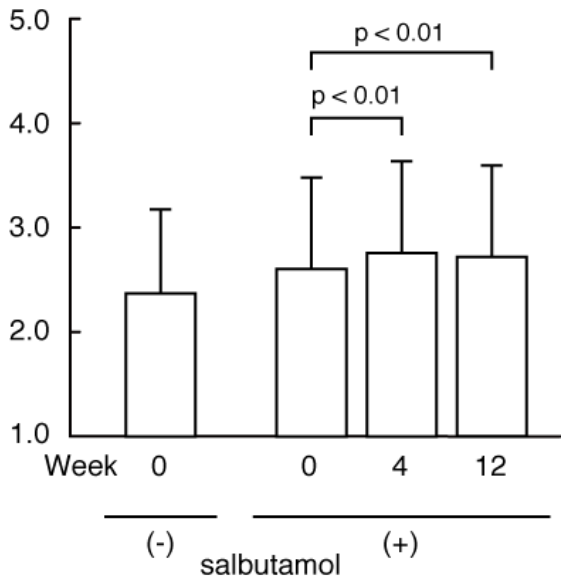

Stages II-IV

$\mathrm{IC}(\mathrm{L})$

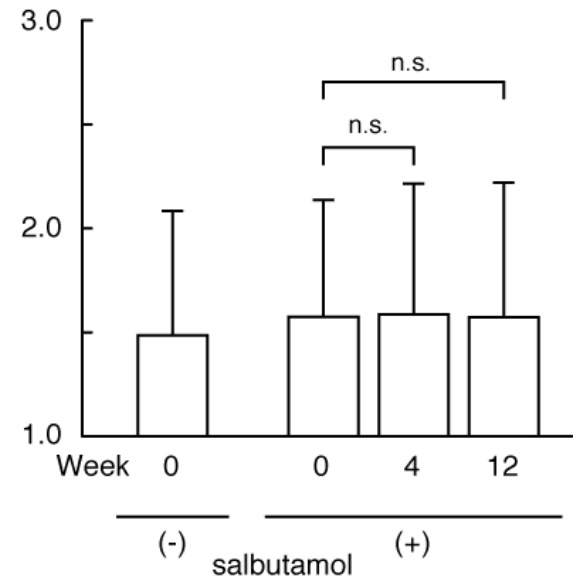

Fig. 1. Pulmonary function test. (A) Values for FEV1, (B) FVC and (C) for IC before and after the tiotropium treatment for the patients who belong to the At risk-Stage I $(n=38)$ and the Stages II-IV $(n=40)$ COPD groups. Error bars indicate standard error. n.s: not significant. 
A

B
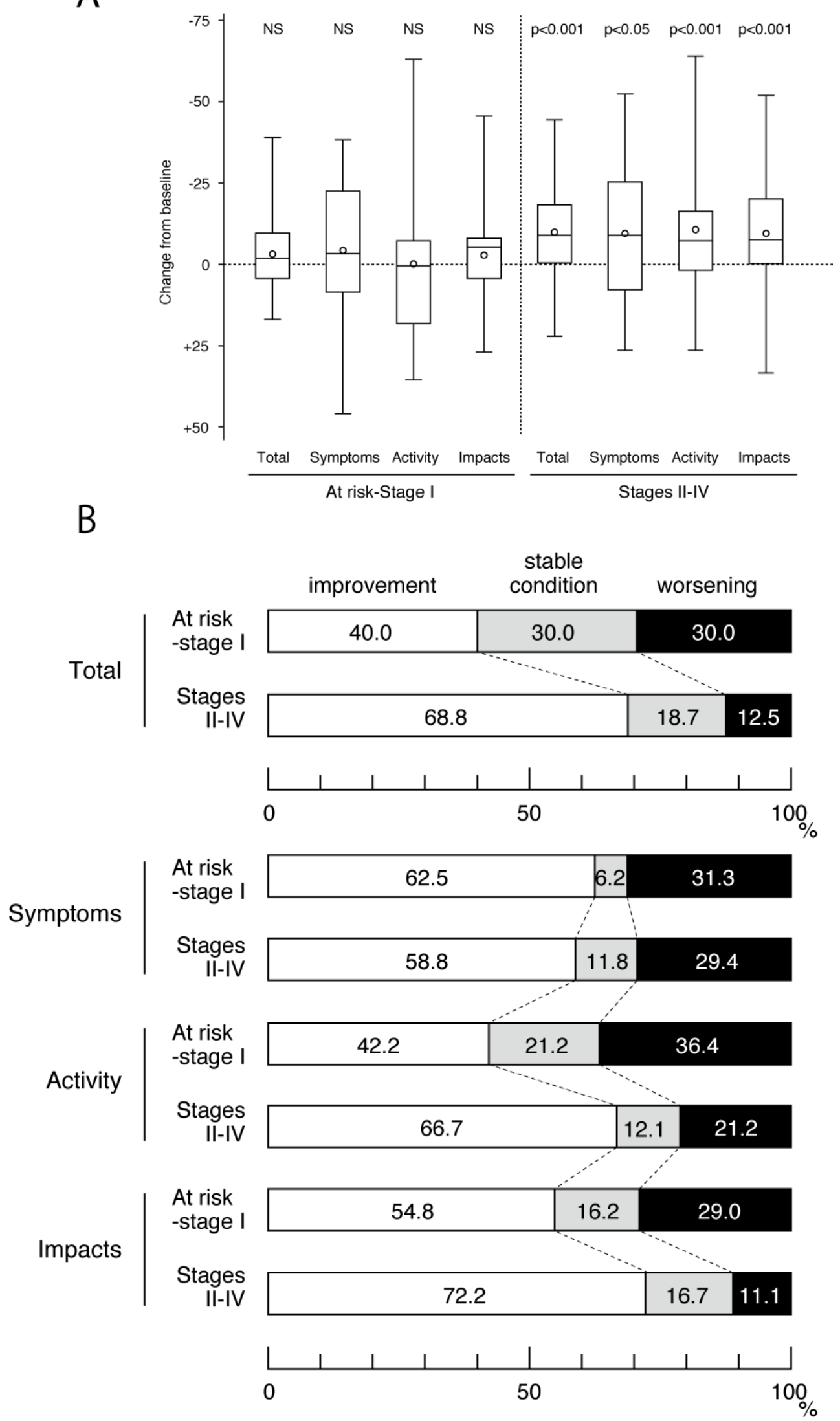

Fig. 2. SGRQ result. (A) A box and whisker plot. A small circle represents the mean, a bar represents the median, a box represents the range for $25-75 \%$ values, and error bars represent the range for $10-90 \%$ values. (B) The proportions of the patients who presented the improvement, worsening and stable disease for the total as well as each domain of the SGRQ are shown. 


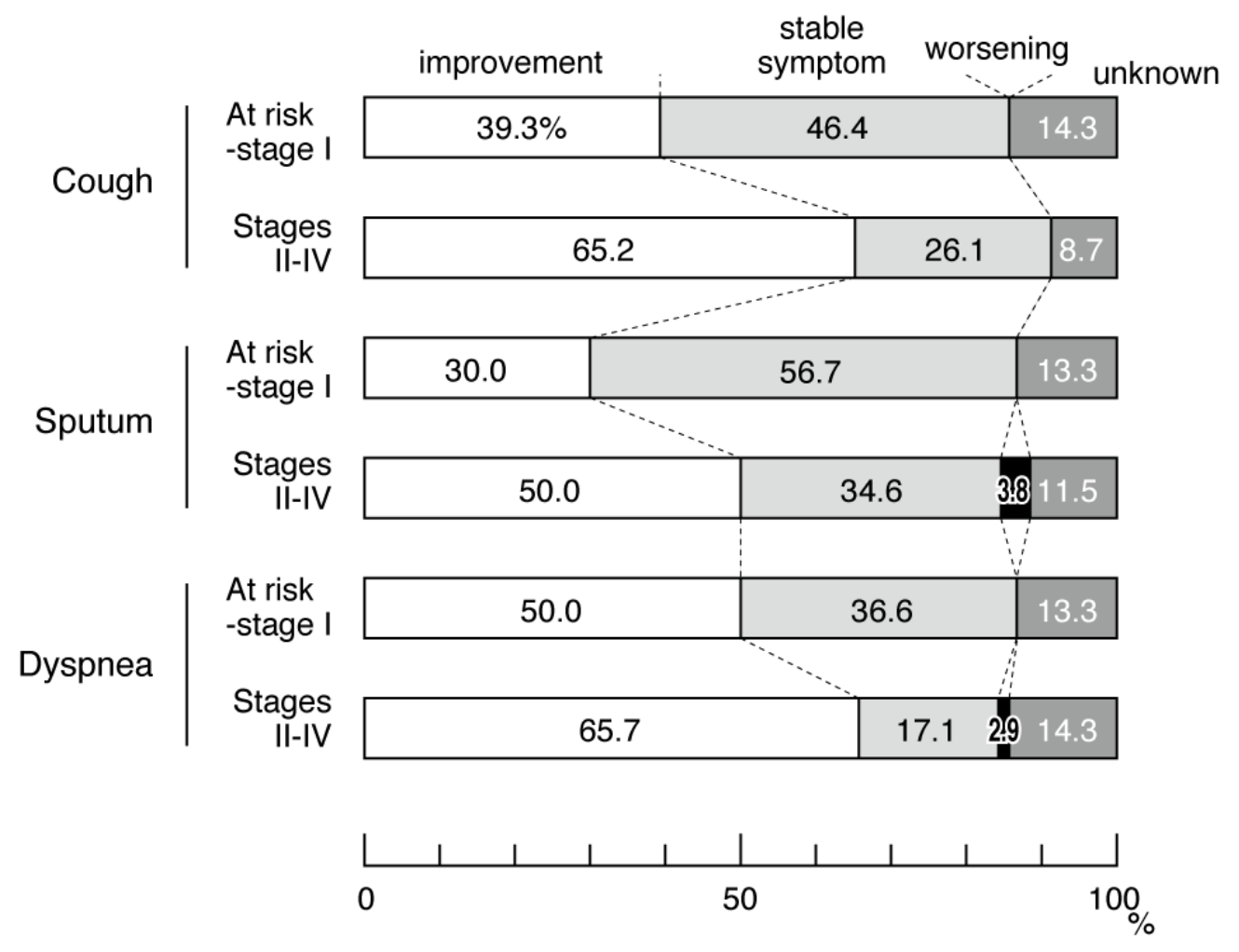

Fig. 3. Change in the clinical symptoms. The proportions of the patients who had an improvement, a worsening and stable symptoms for each group are shown.

current symptoms if they exist. Tiotropium treatment may be effective in this context.

In the current study, we showed that the patients in the At risk-Stage I groups may not benefit from tiotropium treatment either in regard to pulmonary function or in the QOL investigated by the SGRQ. However, symptoms such as cough, sputum and dyspnea may improve in some patients. The result of our study warrants a placebo-controlled study to confirm the effect of tiotropium on the patients with the early stages of COPD.

\section{Acknowledgments}

We thank Drs. Toshiaki Ishii, Isao Kimura, Noboru Kawano, Takashi Matsumoto, Katsuo Ogawa, Masahiko Tanaka, Kazuki Kurashima and Yoshihiro Tanaka from the Saitama Northwest COPD Research Group for their valuable support and advice throughout this study.

\section{References}

1) Celli BR, MacNee W. Standards for the diagnosis and treatment of patients with COPD: a summary of the ATS/ERS position paper. Eur Respir J. 2004; 23: 932-46.

2) Rabe KF, Hurd S, Anzueto A, Barnes PJ, Buist SA, et al. Global strategy for the diagnosis, management, and prevention of chronic obstructive pulmonary disease: GOLD executive summary. Am J Respir Crit Care Med. 2007; 176: 532-55.

3) Mannino DM, Buist AS. Global burden of COPD: risk factors, prevalence, and future trends. Lancet. 2007; 370: 765-73
4) World Health Organization. Revised Global Burden of Disease (GBD) 2002 Estimates: World Health Statistics 2007. 2007.

5) Anzueto A. Clinical course of chronic obstructive pulmonary disease: review of therapeutic interventions. Am J Med. 2006; 119: 46-53.

6) The Japanese Respiratory Society Guidelines for the Diagnosis and Management of COPD, 2nd edition, 2004.

7) Vincken W, van Noord JA, Greefhorst AP, Bantje TA, Kesten S, et al. Improved health outcomes in patients with COPD during 1 yr's treatment with tiotropium. Eur Respir J. 2002; 19: 209-16.

8) Adams SG, Anzueto A, Briggs DD, Jr., Menjoge SS, Kesten S. Tiotropium in COPD patients not previously receiving maintenance respiratory medications. Respir Med. 2006; 100: 1495-503.

9) Niewoehner DE. The impact of severe exacerbations on quality of life and the clinical course of chronic obstructive pulmonary disease. Am J Med. 2006; 119: 38-45.

10) Jones PW, Quirk FH, Baveystock CM, Littlejohns P. A self-complete measure of health status for chronic airflow limitation. The St. George's Respiratory Questionnaire. Am Rev Respir Dis. 1992; 145: 1321-7.

11) Littner MR, Ilowite JS, Tashkin DP, Friedman M, Serby CW, et al. Long-acting bronchodilation with once-daily dosing of tiotropium (Spiriva) in stable chronic obstructive pulmonary disease. Am J Respir Crit Care Med. 2000; 161: 1136-42.

12) Enright PL, Studnicka M, Zielinski J. Spirometory to detect and manage chronic obstructive pulmonary disease and asthma in the primary care setting. In: Grosselink R, Stam H, eds. Lung function testing. European Respiratory Monograph, 2005; 1-14.

13) Jones PW. Interpreting thresholds for a clinically significant change in health status in asthma and COPD. Eur Respir J. 2002; 19: 398404. 